\section{Impact of psychological interventions on reducing anxiety, fear and the need for sedation in children undergoing magnetic resonance imaging}

\author{
Maria Pia Viggiano,1,2 Fiorenza Giganti,1 \\ Arianna Rossi, ${ }^{1}$ Daniele Di Feo, ${ }^{3}$ \\ Laura Vagnoli,2 Giovanna Calcagno, 3 \\ Claudio Defilippi ${ }^{3}$ \\ 1 Department of Neuroscience, \\ Psychology, Drug Research and Child \\ Health, University of Florence; ${ }^{2}$ Pediatric \\ Psychology Service and 3 Radiology \\ Department, Children's Hospital
}

A. Meyer, Florence, Italy

\section{Abstract}

Children undergoing magnetic resonance imaging examination frequently experience anxiety and fear before and during the scanning. The aim of the present study was to assess: i) whether and to what extent psychological interventions might reduce anxiety and fear levels; ii) whether the intervention is related to a decrease in the need for sedation. The interventions consisted of three activities: a clown show, dog interaction and live music. The emotional status (anxiety and fear) of the children was evaluated before and after the activities through a rating scale questionnaire. The results showed that the activities had high effectiveness in reducing the level of anxiety and fear and decreased the need for sedation in the experimental group compared to the control group. This approach proved to be a positive patient experience, helping to alleviate children's anxiety and fear, decreasing the need for sedation, and was cost-effective.

\section{Introduction}

Magnetic resonance imaging (MRI) is an essential diagnostic procedure that has been increasingly used because it provides high quality imaging and does not use ionizing radiation. 1 The procedure is not painful in itself but requires that the patient remain still for approximately 45 minutes.

It must be taken into consideration that some factors related to MRI procedure (noises, unfamiliar environment, presence of unknown staff) can cause anxiety, nervousness, fear and agitation in patients undergoing MRI.2 Children are especially prone to these anxi- eties and therefore sedation is sometimes used in an attempt to help them to keep still. Although it is well known that sedation has the disadvantage of increasing i) the patient's risk (e.g., respiratory depression and troubles of the cardiac rhythm, with an incidence of 1/10.000); moreover, some of types of sedation (e.g., using the hydrated cloralium) could cause psicomotor nervousness or drowsiness extended for over 6 hours in 1/3 of the cases (Pediatric Anesthesia and Critical Care Journal, 2007); ii) health care costs (e.g., supplementary staff MRI-compatible equipment, drugs and increased preparation time), reliable images are often obtained exclusively under deep sedation. ${ }^{3-7}$ However, to decrease the need of sedation, distress, nervousness and anxiety in children undergoing MRI, several interventions have been adopted. ${ }^{8}$ For example, Carter et al. ${ }^{1}$ performed a mock MRI before the true scan, and reported a benefit consisting in decreased sedation in children ranging from 3 to 8 years. Encouraging results were also obtained with the implementation of an Audio/Visual (AV) system that allowed children to watch a video within the MRI magnetic field.9,10 They found that the need of sedation significantly decreased (from $49 \%$ to $40 \%$ ) in children undergoing MRI. Along the same lines, Lemaire et al.,11 with the implementation of the AV system, showed a decrease in sedation of $34 \%$ in children ranging from 4 - to 10 -year old, whereas the values did not reach the significance for the remaining age groups. Other types of interventions such as watching a movie showing MRI procedures, breathing exercises, listening to music and guided imagery, have been shown to have overall, although lower effectiveness.12,13 Train et al.14 found that psychological preparation through both a photo-booklet and written advice to parents led to reduced anxiety and decreased rates of sedation. The effect of a photo diary was also investigated by Hartman et al.15 They found no difference in terms of anxiety reduction between children to whom an illustrated booklet containing detailed information on the MRI procedure was provided and the control group (to whom only standard information was delivered). The above mentioned studies indicate a real need to adopt interventions and strategies to decrease both anxiety and the use of sedation in children undergoing MRI. It is worth noting that a crucial moment of increased anxiety, in both children and parents, may occur in the waiting room, waiting for the examination. In line with a need to decrease anxiety, the present study has twofold aims: i) to assess whether and to what extent psychological interventions might modulate anxiety and fear in children aged 4 to 11 years; ii) to verify whether the psychological intervention is related to a reduced need for sedation.
Correspondence: Maria Pia Viggiano, Department of Neurosciences, Psychology, Drug Research, Child Health, Via San Salvi 12, 50135 Florence, Italy.

Tel.: +39.055.2755.053 - Fax: +39.055.623.6047 E-mail: mariapia.viggiano@unifi.it

Key words: magnetic resonance imaging, sedation, psychological interventions, anxiety, fear.

Acknowledgements: we are grateful to Anna Meyer Foundation which supporting "Incontri con gli animali", "Clown in Corsia" and "Musica in corsia" projects; the professionals of Antropozoa onlus, the clowns of Soccorso Clown onlus, the musicians of Athenaeum Musicale Fiorentino and the health professionals of Radiology Department who work in Children's Hospital A. Meyer, Florence, Italy.

Contributions: CD, MPV, integrity of the entire study; MPV, CD, FG, LV, study concepts and design; AR, MPV, FG, literature research; AR, LV, DDF, GC, experimental studies and data analysis; MPV, FG, statistical analysis; CD, MPV, FG, manuscript preparation; MPV, LV, DDF, GC, manuscript editing.

Conflict of interest: the authors declare no potential conflict of interest.

Received for publication: 16 October 2014. Accepted for publication: 22 January 2015.

This work is licensed under a Creative Commons Attribution NonCommercial 3.0 License (CC BYNC 3.0).

(C) Copyright M.P. Viggiano et al., 2015

Licensee PAGEPress, Italy

Pediatric Reports 2015; 7:5682

doi:10.4081/pr.2015.5682

For this purpose, we organized three different activities before the MRI session, consisting of live music, presence of hospital clown and animal assisted activities. The beneficial effect of these interventions has been documented also in a more extensive context. These approaches might act not only in reducing anxiety and fear in children (through direct interaction with music, clowns and dogs) but also in creating a general relaxed atmosphere involving parents and medical staff, with a positive feedback effect on children.16-19 In this way the child is doubly benefited. A questionnaire based on a rating scale was designed to capture changes in emotions before and after the activities.

\section{Materials and Methods}

\section{Subjects}

The subject groups were recruited from the pediatric patients referred to the Meyer Children's Hospital, Florence, Italy, for MRI examinations. Subjects were randomly 
assigned to the experimental or control groups. Forty children (21 males) between 4 and 11 year-old were enrolled in the experimental group and 65 children (30 males) aged between 4 and 11 years in the control group. The demographic and clinical characteristics of the two groups of children are reported in Table 1. The inclusion criteria for the patients were: i) first attempt of examination without sedation; ii) duration esteemed of the examination less than 60 minutes.

\section{Procedure}

Parents were explained the procedure and informed consent was obtained.

Children included in the experimental group were asked before the MRI examination to indicate on a 5-point Likert scale their emotional status regarding anxiety and fear. A cartoon figure representing different degrees of anxiety and fear represented each point from the lowest degree to the highest one. After children completed the two scales, staff conducting different interactions came close to the patients and started the activity for 10 minutes. Three different activities were proposed: i) clown (two professional clowns interacted with children by telling jokes or funny stories; ii) animal assisted intervention (children interacted with a dog, petting or giving a cookie, under the supervision of a professional trainer); iii) live music (a professional musician played an instrument (violin, flute) in front of the child proposing him to play other instruments appositely created for children). Each patient was randomly exposed to only one of these activities. In the experimental group 15 children interacted with clown, 12 children with dogs and 13 children with musicians After the activity, children were again asked to indicate their emotional status regarding anxiety and fear. After children completed the two scales, the MRI examination started.

Patients included in the control group were asked, before the MRI examination, to indicate on a 5-point Likert scale their emotional status regarding anxiety and fear, after about $20 \mathrm{~min}$ utes, they were scanned.

\section{Statistical analysis}

The SPSS (Statistical Package for the Social Science) for Windows was used for statistical analysis. For the comparison of categorical data, the chi-square test was used, and for the comparison of numerical data, Student's t-test was used. The emotional status of experimental group patients before the activities was compared to that of control group patients before the MRI examination. This analysis was performed to ascertain that experimental and control groups did not differ in their baseline emotional status.

To evaluate the effectiveness of psychological activities in modifying the emotional status of the subjects, the levels of anxiety and fear before and after the activities were compared. To evaluate the effect of activities on the need for sedation, patients requiring sedation and patients not requiring sedation in experimental and control groups were entered in a binary logistic regression with age and activity as covariates. This statistical model was used because the independent variable (sedation) was dichotomous and the independent variables (activity and age) were categorical and numerical respectively. Significance was set at $\mathrm{P} \leq 0.05$.

Table 1. The children's parameters expressed as mean \pm standard deviation (SD).

\begin{tabular}{lccc} 
Parameters & Experimental group & Control group & P \\
Age & $7.8 \pm 1.7$ & $8 \pm 1.7$ & 0.61 \\
Gender & & & 0.52 \\
$\quad$ Male, $\mathrm{n}$ & 21 & 30 & \\
$\quad$ Female, $\mathrm{n}$ & 19 & 35 & 0.88 \\
\hline Duration of MRI (min) & $48.7 \pm 15.1$ & $48.3 \pm 15.6$ & 0.75 \\
MRI-body part imaged & 34 & 57 & \\
$\quad$ Brain & 3 & 4 & \\
Columna vertebralis & 2 & 1 & \\
Abdomen & 1 & 3 & \\
Extremity-joint & & & \\
\hline
\end{tabular}

MRI, magnetic resonance imaging.

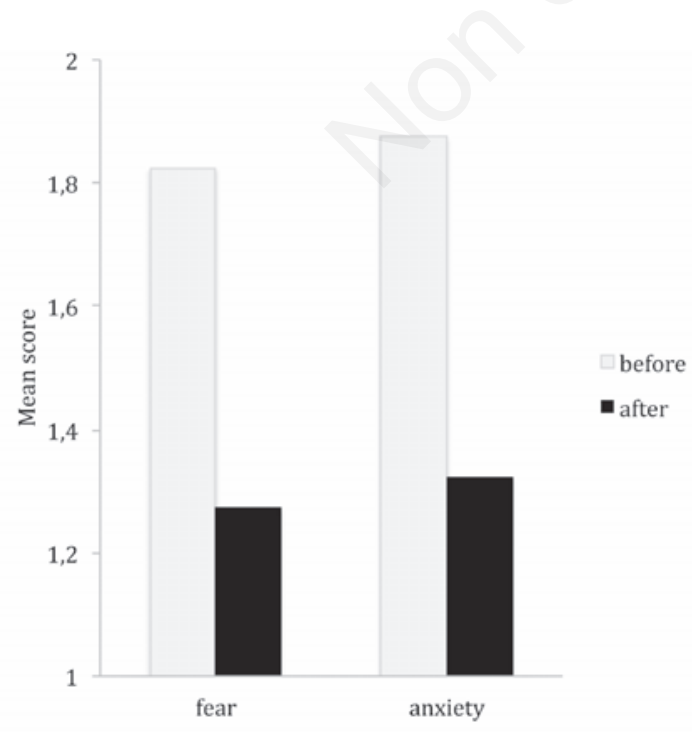

Figure 1. Average scoring, for fear and anxiety, obtained by experimental group before and after the psychological intervention.

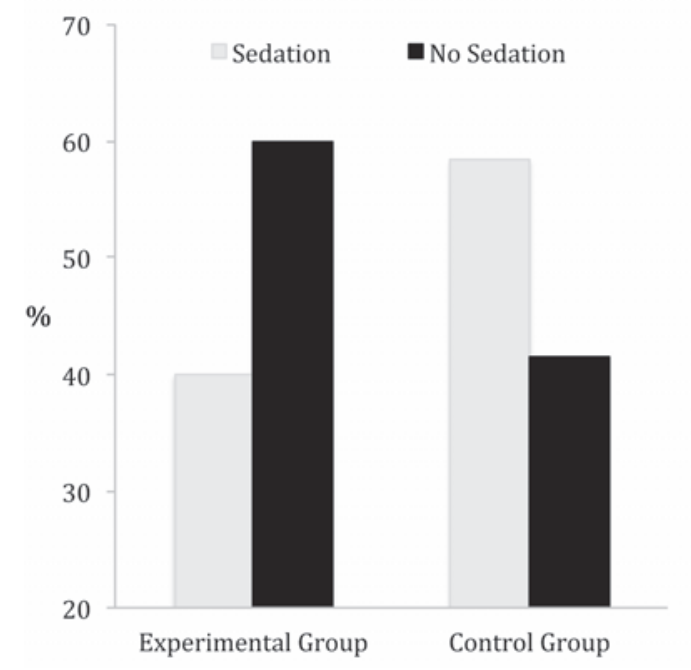

Figure 2. Percentage of pediatric patients who had (experimental group) or had not (control group) partecipated to activities. A significant $(P<0.025)$ decreasing in the need for sedation emerged for experimental group. 


\section{Results}

Table 1 shows children's demographic data. There were no significant differences between the experimental and control groups in terms of age, gender and duration of their MRI tests. The most frequently imaged organ was the brain without significant differences between the two groups.

In the experimental group, the emotional status evaluated before the activities was not significantly different from that in the control group prior to MRI examination. In addition, in the experimental group, the activities had a beneficial effect on the patients' emotional status with a decreasing of both anxiety and fear level $(\mathrm{P}<0.01$ and $\mathrm{P}<0.001$ respectively; Figure 1).

Finally, from the binary logistic regression, results indicate that the effect of activity experience on need for sedation was statistically significant $(\mathrm{P}<0.025)$. The need for sedation was less in those children who engaged in one of the activities compared to the control group (Figure 2). Furthermore, the main effect of age was found $(\mathrm{P}<0.001)$ : a decreasing of need for sedation was observed to a greater extent in older children compare with younger ones.

\section{Discussion and Conclusions}

Children undergoing MRI examination frequently experience negative emotions. The unknown staff, noises and unfamiliar environment can provoke anxiety and fear and as consequence they cannot remain still during the procedure. Since obtaining a motion-free MRI scan can be a challenge in children, sedation is sometimes necessary.

The present study was designed to evaluate the effectiveness of psychological interventions in counteracting anxiety and fear and to assess whether such interventions might decrease the need for sedation. Our results show that such interventions can have a beneficial effect by reducing negative emotions.

In addition, we found a reverse relationship between participation in a psychological activity and the need for sedation. Children belonging to the experimental group had significantly less need of sedation. The data show a decrease over about 3 months in the number of children requiring sedation to complete their MRI examination. As shown in Figure 2, the need for sedation decreased of $18 \%$ in the experimental group compared to the control group.

We might hypothesize that the activities acted as distractions from the anxiety-provok- ing stimulus and, at the same time, created a general relaxing atmosphere that eased the tension of both children and their parents. We also suppose, although only in a speculative way, that the relaxing atmosphere created by the activities in the waiting area also affected the mood of the MRI staff, thus improving their interactions with children and their families. This appears an important issue and could warrant further investigations

It is noteworthy that scarce attention has been devoted to this topic; for this reason it is difficult to compare the effectiveness of different approaches through only a few previous studies. However, it seems that the different strategies proposed in these studies have had overall positive affects. Nevertheless, it should be taken into account that the interventions adopted in the present study are difficult to organize when urgent basis examinations are required. However, it might be a useful approach to combat anxiety in scheduled examination conditions.

Summing up, our present findings indicate that the psychological interventions (clown show, dog interaction and live music) were beneficial in helping to alleviate children's anxiety and fear. Although by no means conclusive, they constitute further confirmation that it is possible to modulate negative emotions and reduce rates of sedation in children undergoing MRI scan.

\section{References}

1. Carter AJ, Greer MLC, Gray SE, Ware RS. Mock MRI: reducing the need for anaesthesia in children. Pediatr Radiol 2010;40:1368-74.

2. Munn Z, Jordan Z. The patient experience of high technology medical imaging: a systematic review of the qualitative evidence. Radiography 2011;17:323-31.

3. Formica D, Silvestri S. Biological effects of exposure to magnetic resonance imaging: an overview. BioMed Eng Online 2004;3:1475-95.

4. Voepel-Lewis T, Malviya S, Prochaska G, Tait AR. Sedation failures in children undergoing MRI and CT: is temperament a factor? Paediatr Anaesth 2000;10:319-23.

5. Weissend EE, Litman RS. Paediatric anaesthesia outside the operating room. Curr Opin Anaesthesiol 2001;14:437-40.

6. Gooden CK. Anesthesia for magnetic resonance imaging. Curr Opin Anaesthesiol 2004;17:33-42.

7. Haeseler G, Zuzan 0, Köhn G, et al. Anaesthesia with midazolam and S-(+)ketamine in spontaneously breathing pae- diatric patients during magnetic resonance imaging. Paediatr Anaesth 2000;10:513-9.

8. Munn Z, Jordan Z. Interventions to reduce anxiety, distress, and the need for sedation in pediatric patients undergoing magnetic resonance imaging: a systematic review. J Radiol Nurs 2013;32:87-96.

9. Herned IRK, Strain JD. MRI-compatible audio/visual system: impact on pediatric sedation. Pediatr Radiol 2001;32:247-50.

10. Tornqvist E, Masson A, Hallstrom I. Children having magnetic resonance imaging: a preparatory storybook and audio/visual media are preferable to anesthesia or deep sedation. J Child Health Care 2014 [Epub ahead of print].

11. Lemaire C, Moran GR, Swan H. Impact of audio/visual systems on pediatric sedation in magnetic resonance imaging. J Magn Reson Imaging 2009;30:649-55.

12. Smart G. Helping children relax during magnetic resonance imaging. MCN Am Matern Child Nurs 1997;22:236-41.

13. Tyc VL, Leigh L, Mulhern RK, et al. Evaluation of a cognitive-behavioral interventions for reducing distress in pediatric cancer patients undergoing magnetic resonance imaging procedures. Int J Rehabil Health 1997;3:267-79.

14. Train H, Colville G, Allan R, Thurlbeck S. Pediatric 99mTc-DMSA imaging: reducing distress and rate of sedation using a psychological approach. Clin Radiol 2006;62: 868-74.

15. Hartman JH, Bena J, McIntyre S, Albert NM. Does a photo diary decrease stress and anxiety in children undergoing magnetic resonance imaging? A randomized, controlled study. J Radiol Nurs 2009;28: 122-28.

16. Yip P, Middleton P, Cyna AM, Carlyle AV. Non-pharmacological interventions for assisting the induction of anaesthesia in children. Cochrane Database Syst Rev 2009:CD006447.

17. Vagnoli L, Caprilli S, Robiglio A, Messeri A. Clown doctors as a treatment for preoperative anxiety in children: a randomized, prospective study. Pediatrics 2005;116: 563-7.

18. Balan R, Bavdekar SB, Jadhav S. Can indian classical instrumental music reduce pain felt during venipuncture? Indian $\mathrm{J}$ Pediatr 2009;76:469-73.

19. Dietz TJ, Davis D, Pennings J. Evaluating animal-assisted therapy in group treatment for child abuse. J Child Sex Abus 2012;21:665-83. 